\title{
Craniosynostosis - A Case Series and a Brief Review of Literature
}

\author{
Sibhi Ganapathy ${ }^{1, \text { *, Swaroop Gopal }}{ }^{2}$ \\ ${ }^{1}$ Manipal Institute of Neurological Disorders (MIND), Bangalore, India \\ ${ }^{2}$ Institute of Neurosciences, Sakra World Hospital, Bangalore, India
}

Email address:

sibhig09@gmail.com (S. Ganapathy), Swaroop.gopal@sakrahospital.com (S. Gopal)

${ }^{*}$ Corresponding author

To cite this article:

Sibhi Ganapathy, Swaroop Gopal. Craniosynostosis - A Case Series and a Brief Review of Literature. Clinical Neurology and Neuroscience. Vol. 4, No. 2, 2020, pp. 38-43. doi: 10.11648/j.cnn.20200402.14

Received: May 16, 2020; Accepted: May 29, 2020; Published: June 28, 2020

\begin{abstract}
Introduction: Craniosynostosis is an uncommon disorder affecting the growing skull. Timely surgery and physical therapy can give excellent results restoring neurological function and cosmesis. However, miss the appropriate juncture, and severe consequences will follow. Concerns include late presentation, surgical morbidity. Case Series: We present our institutional experience of varied syndromes who presented to a tertiary care institution between 2016 and 2018 along with the course in hospital complete with surgery and rehabilitation. We also supplement this with a short review of literature. The article stresses on the need to differentiate syndromic and simple craniosynostosis as well as in their specific management strategies complete with procedure assessment and complications. Result: A series of syndromic and simple craniosynostosis operated early lead to optimal cosmetic results with minimal or no long-term neurological deficits. The approach emphasises the need for early treatment to ensure excellent cosmesis and to avoid neurological and developmental disorders.
\end{abstract}

Keywords: Craniosynostosis, Strip Craniotomy, Suture Excision

\section{Introduction}

Craniosynostosis is defined as a premature closure of one or more of the cranial sutures leading to raised intracranial pressure and developmental disorders of the brain. The incidence and occurrence are sporadic with certain recognised risk factors. Occasionally, syndromic craniosynostosis also exists, where a combination of cranial and extracranial abnormalities give rise to a complex set of problems often difficult to handle. We present a simple summary of the disease data from our institution, collected over 5 years with patients followed up for 5 years post-surgery. The results, and inferences gleaned from these patients is presented below.

Primary craniosynostosis: a primary defect of ossification.

\subsection{Suture Lines Across the Skull are Described Below}

Metopic Suture: This exists between the 2 growing frontal bones. It closes by 3-9 months of age. It exists to allow the growth of the massive frontal lobes responsible for personality, emotion, cognition and speech.
Coronal Suture: This is the next most commonly affected suture by craniosynostosis. It fuses normally by 24years of age completely. Coronal suture allows the growth of the parietal and temporal lobes of the brain.

Sagittal Suture: The longest and most important suture present fuses by the $3^{\text {rd }}$ decade of life. It allows the complete development of the hemisphere. The anterior fontanelle, the point of convergence of the coronal, metopic and sagittal sutures closes by 18 months of age. A bulging Anterior Fontanelle (AF) is generally a sign of raised ICP, and requires investigation.

Lambdoid Suture: This occurs most commonly as a consequence of not moving the child from the bed, leading to a positional pressure related deformity. Usually if unilateral they result in occipital plagiocephaly. If bilateral, (rarely) it results in a tower skull deformity (especially if associated with coronal synostosis as well) known as Turricephaly or Oxycephaly.

\subsection{Craniosynostosis Is Classified as Either}

Primary (When 1 or more sutures fuse prematurely, skull 
growth can be restricted perpendicular to the suture. If multiple sutures fuse while the brain is still increasing in size, intracranial pressure can increase. The cause has been identified as a primary defect in the mesenchymal layer ossification in the cranial bones. A gene locus for single suture craniosynostosis has not been identified.

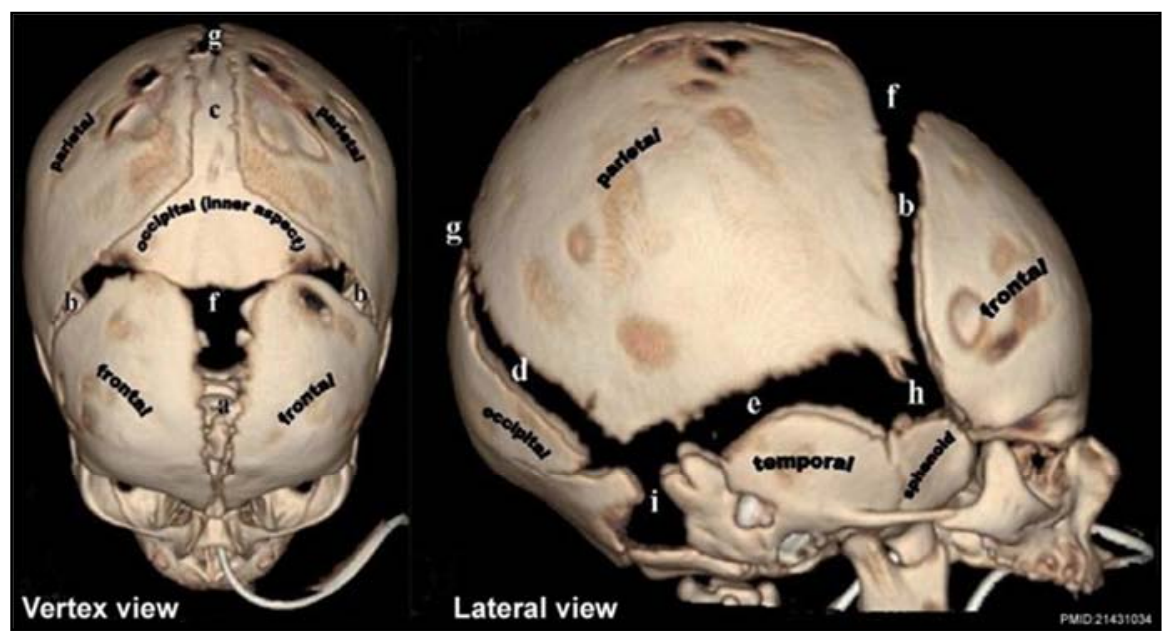

Figure 1. Showing sutures of the skull.

Secondary craniosynostosis is described as a failure of brain growth, which precipitates a small and often misshapen skull. This is less common. The disease can also be classified as Simple craniosynostosis, where only 1 suture fuses prematurely or Complex or compound craniosynostosis, where premature fusion of multiple sutures occurs. The primary factor that keeps sutures open is ongoing brain growth. Normal skull growth occurs perpendicular to each suture. The suture lines aren't fused at birth to enable the skull to be flexible enough to get through vagina. This phenomenon is called moulding. The skull as a whole however needs to be expansile enough to accommodate rapid brain growth.

Syndromic Craniosynostosis Although only 10-20\% of cases, these conditions form a complex series of disorders which make successful resolution difficult. They are usually autosomal Dominant in inheritance linked to Chromosome 10q. Syndromic craniosynostosis are usually multi-sutural, \& complex cases requiring a multidisciplinary approach along with long term care in order to achieve good results..

Table 1. Distinguishing features of syndromic craniosynostosis.

\begin{tabular}{lllll}
\hline & Muenke & Crouzon & Jackson-Weiss & Apert \\
\hline Thumbs & Normal & Normal & - & Fused to fingers \\
Hands & Carpal fusion & Normal & Variable & Broad \& Deviated \\
Great Toe & Broad & Normal & Broad \& deviated & Bone Syndactyly \\
Feet & Tarsal fusion & Normal & Abnormal tarsals & Fused to toes \\
\hline
\end{tabular}

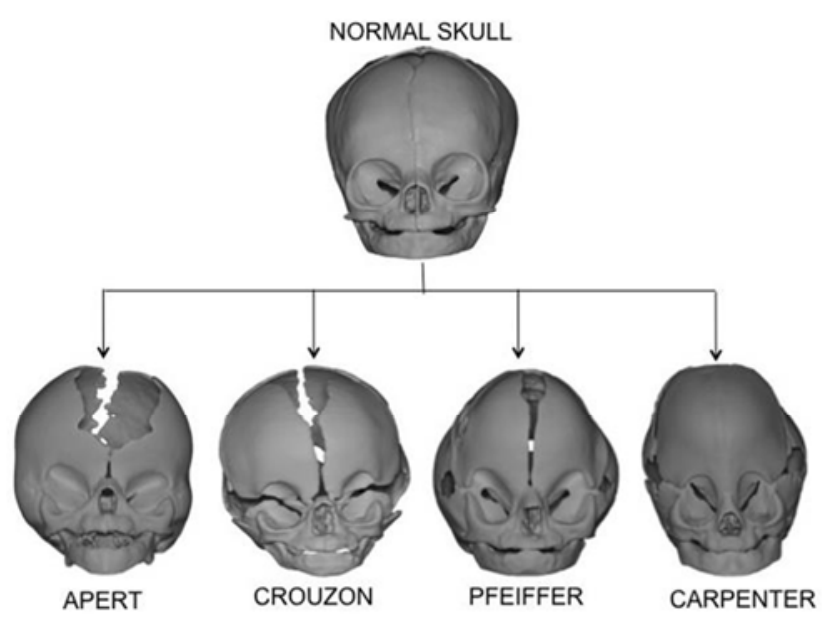

Figure 2. Skull deformity in syndromic craniosynostosis.

Diagnosis is made clinically through analysis the shape of skull, detecting features of raised ICP, documenting delayed developmental milestones and assessing syndromic features in other organ systems (in complex synostoses) confirmation is usually made through imaging modalities. A summary of the common syndromes resulting in craniosynostosis are listed in Table 1.

\section{Common Clinical Presentations Include}

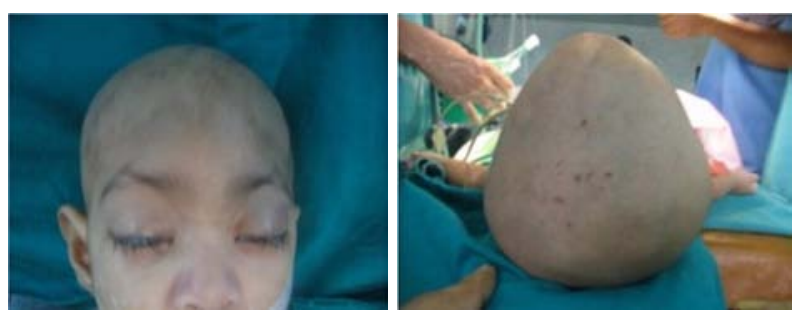

Figure 3. Trigonocephaly. 

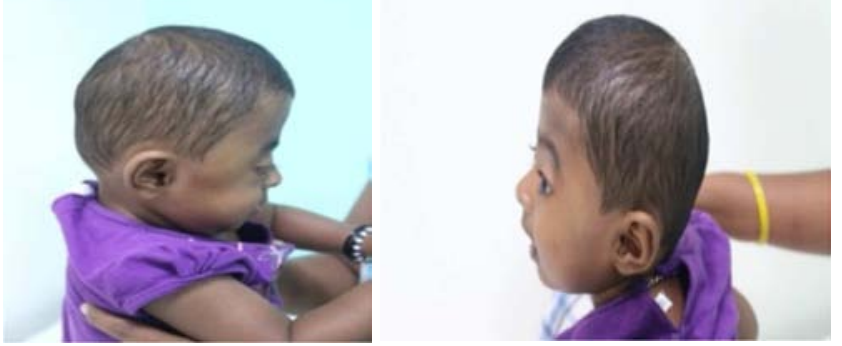

Figure 4. Brachycephaly.

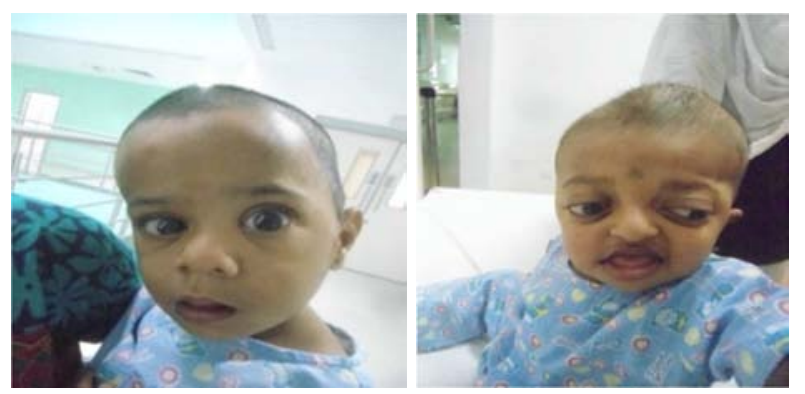

Figure 5. Anterior Plagiocephaly.
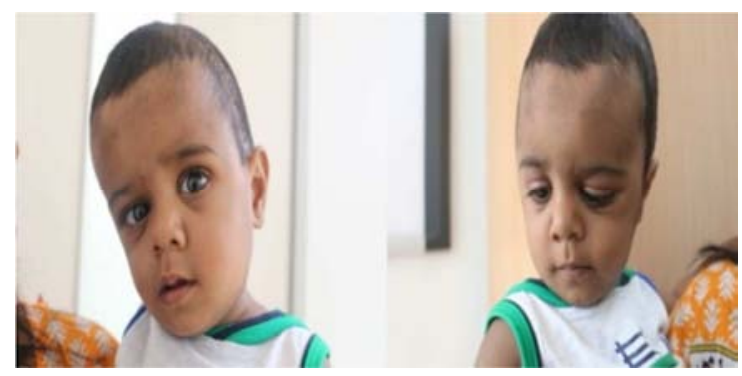

Figure 6. Dolichocephaly.

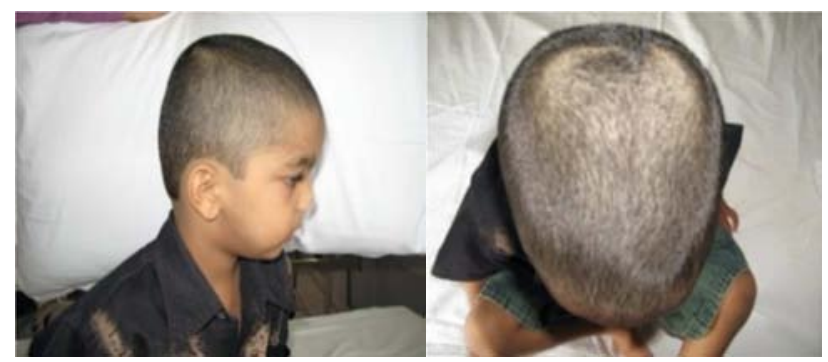

Figure 7. Occipital Plagiocephaly.

\section{Radiology}

$\mathrm{X}$-Ray of the skull is the best screening modality, where one looks for a copper beaten appearance along with premature fused sutures. Once suspected, further investigations are done with CT scans of the head. These are the best for pre-op planning ( $3 \mathrm{~d}$ recon imaging) especially in craniofacial abnormalities with syndromic craniosynostosis. MRI brain is useful only in patients with developmental delay, seizures, suspected hydrocephalus or features of ICP. MRI screening of the brain is mandatory in syndromic craniosynostosis. The Decision to Operate depends upon the presence of features of raised ICP along with any neurological deficit. Particular importance is given to vision, speech and gait above others due to their vital importance in further brain development coupled with the poor prognosis in recovery once damaged. Learning regression (especially in association with other deficits) is also an important indication when clearly documented. Cosmetic considerations usually most important as it affects peer acceptance, parent-child bonding, self-image and coping.

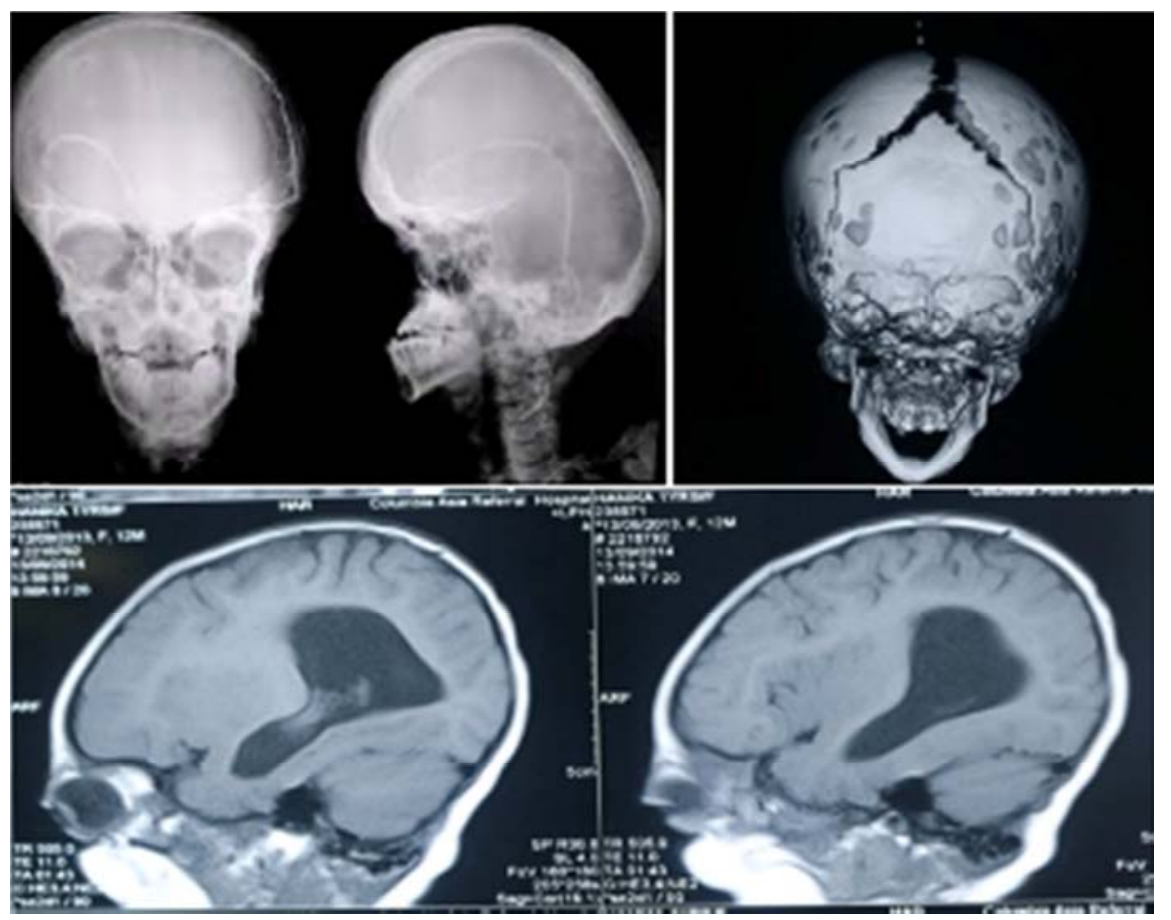

Figure 8. Radiological features of craiosynstosis consisting of Xray images (A) showing deformed shape with copper beaten appearance, (B) CT skull showing the $3 D$ recon of the skull, and (C) MRI of the brain showing hydrocephalus and a mishapen ventricle. 


\section{Surgical Correction}

Surgical correction once decided upon can assume many forms ranging from distractor placement to the time and tested strip craniotomy concept. Surgery for craniosynostosis has undergone a massive improvement over the last decade, with a spectrum of procedures available to suit different situations thereby reducing the morbidity of surgery and improving post-surgery. Types of surgery range from:

\subsection{Suture Excision}

Here there is cutting of the fused suture allowing normal brain expansion to occur. It can be done endoscopically with small incisions, thereby reducing blood loss and effect early discharge (2-3 days alone) A 30-degree endoscope is used through a remote incision and bone cuts are made by strong scissors followed by removal of fused bone and placement of the $3 \mathrm{D}$ printed helmet.
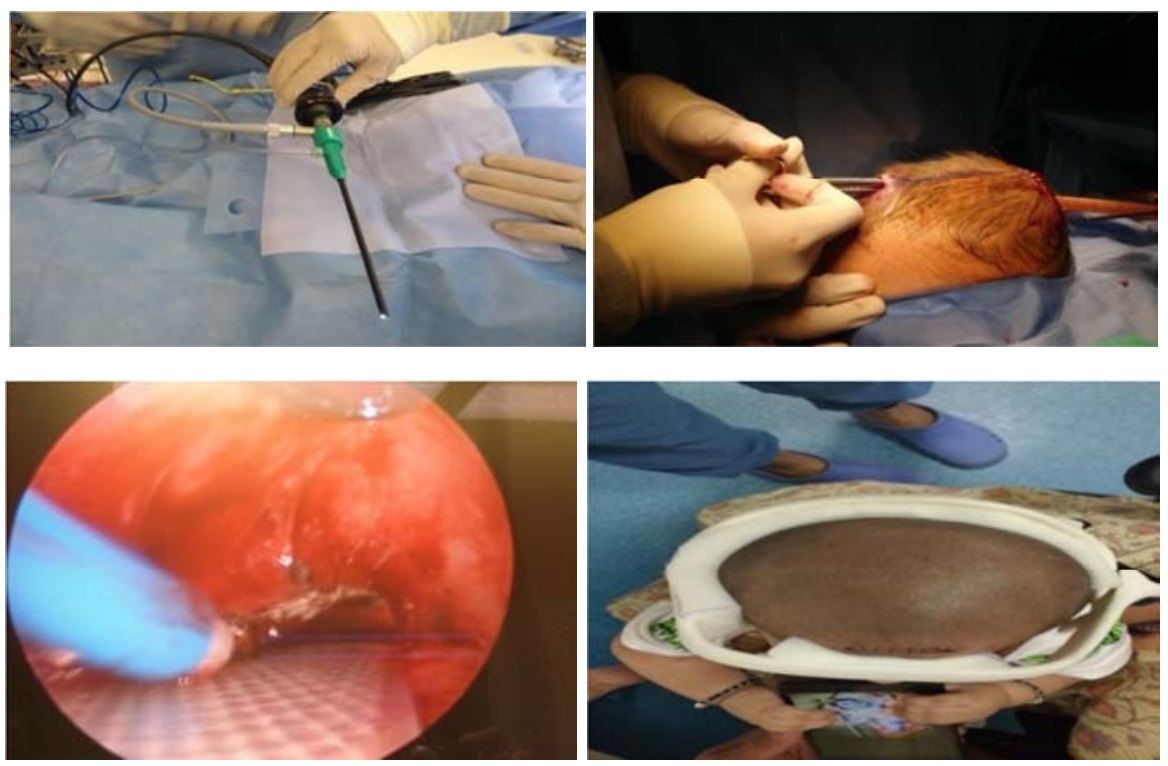

Figure 9. Endoscopic suture excision done using a 30degrees endoscope (A), being used to cut the bone with a scissors (B), the suture bone being removed (C) and the child given a specially designed helmet to preserve the contour of the skull. (D).

\subsection{Strip Craniotomy}

The commonest and most successful procedure done is the strip craniotomy. It is ideally performed in young infants less than $2 \mathrm{yrs}$ of age, and only if brain growth is stifled.
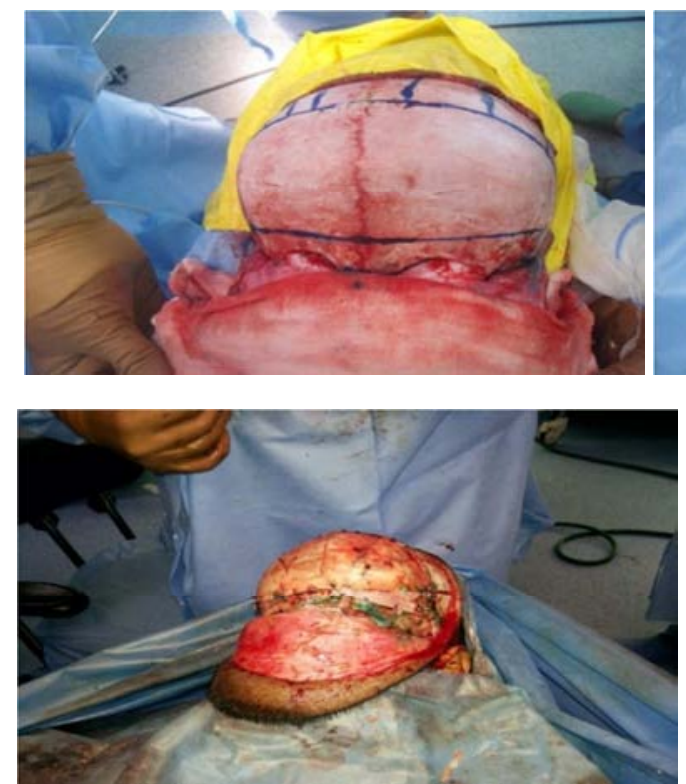

Sometimes skin closure and healing can be challenging. recovery and cosmesis. After surgery a special helmet is placed over the head to ensure moulding of the now mobile skull strips.
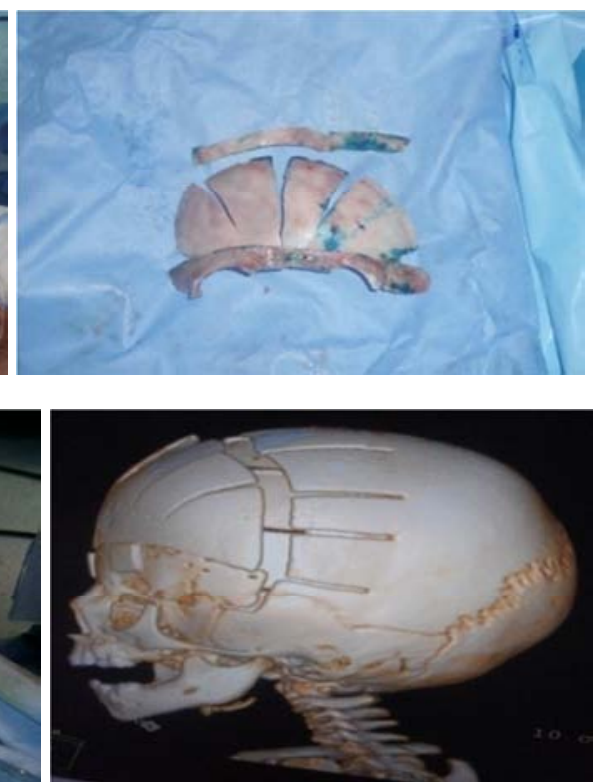

Figure 10. Bicoronal flap with strips marked on the skull (A), the bone removed and cut into strips (B), and repositioned back to the skull (C) The Post Op CT scan showing the strip craniotomy reshaping the skull. 


\subsection{Orbital Advancement}

Orbital advancement the commonest procedure for facial dysmorphisms especially in syndromic craniosynostosis. It combined with strip craniotomies offers excellent results. It is usually done usually after 2 years of age. Here bone pieces cut, reshaped and put back along with a remodelled skull. A CT scan after surgery confirms the results.
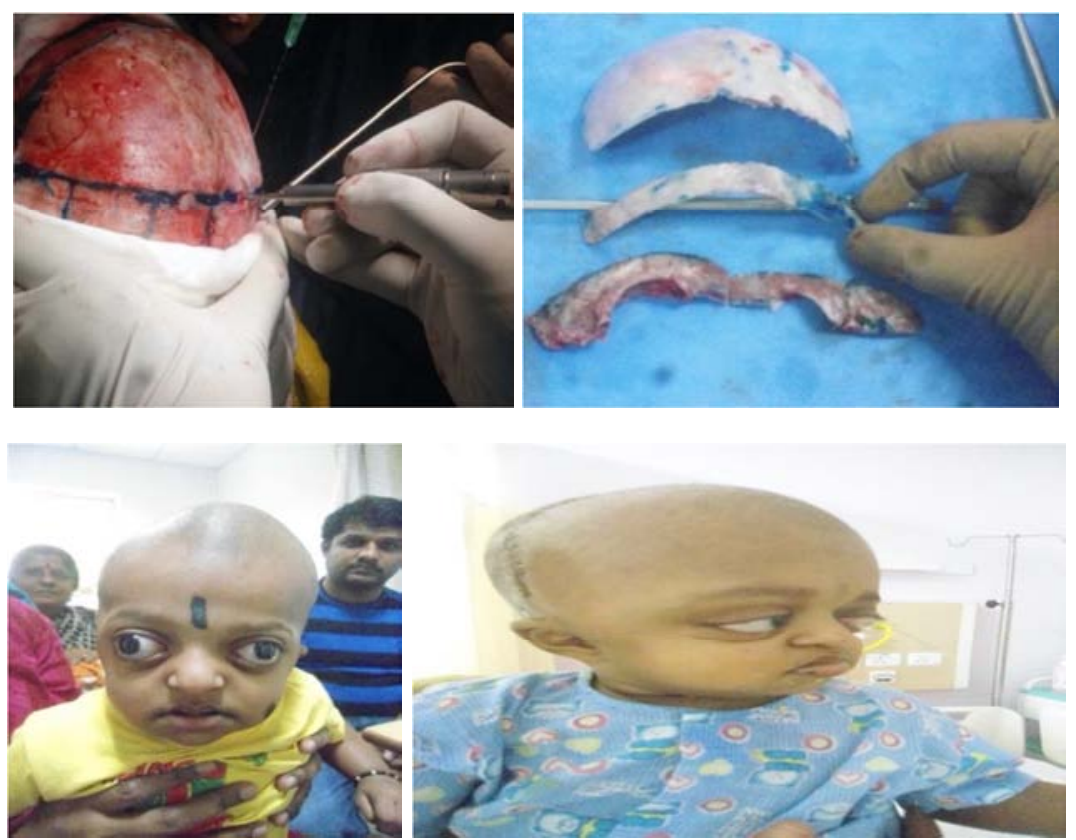

Figure 11. burrholes made to start the craniotomy (A), with removal of the frontal bones and orbittal ridge as seen in (B), with the before surgery (C), and post op images (D).

Complications of Surgery include blood loss, hypothermia, post-operative seizures, CSF leak, wound infection, meningitis, non-healing of sutures, implant failure (if using distractors) There may be a need for multiple procedures as well in the future. Impairment of ocular mobility, dissociated movements, amblyopia and refractive errors. Pre- and postop impairments seen most frequently with unilateral coronal and metopic synostoses. Thus, long term follow-up remains vital to ensuring the gains of surgery do not gradually end up lost. Follow up must be done regularly upto 12 years (until bone maturity develops) with special emphasis given to vision, speech, feeding \& swallowing. Lastly genetic counselling is vital for compliance of the family to potentially distressing therapy and surgery in very young children.

\subsection{Certain Special Conditions May Mimic Craniosynostosis But May Not Need Surgery}

These conditions include:

VP shunting cause scaphocephaly, and chronic hydrocephalus thickening the skull. In such conditions surgical Indications are definitive. Here an OFC (Occipital Frontal Circumference) $>50 \mathrm{~cm}$ (4-5+ STDs), along with when VP shunt performed on very low birth weight babies.

Prematurity, leading to deformational scaphocephaly associated with impaired mobility due to prolonged positioning. If not corrected, it may persist until adulthood. Prevention of craniosynostosis can be effected by using donut-shaped head supports, and waterbed mattresses. Most conditions do not warrant intervention.

Microcephaly. Here surgical correction not indicated despite an abnormal OFC as in primary craniosynostosis, OFC remains normal yet oddly shaped. These are rare cases of multisutural craniosynostosis restricting head growth, but which manifest with increased ICP.

Positional Deformation. This is the most common cause of an abnormal skull shape. Usually only forehead asymmetry occurs sometimes associated with torticollis. Coronal or lamboidal sutures maybe involved as well. Around $40 \%$ of new-borns.

\section{Conclusion}

Craniosynostosis is treatable with minimally invasive surgery tailored to suit the needs of each individual patient. Proper follow up and counselling give rise to excellent longterm results with prevention of brain damage.

\section{References}

[1] Ocal E, Sun PP, Persing JA. Craniosynostosis. In: Albright AL, Pollack IF, Adelson PD, editors. Principle and practice of pediatric neurosurgery. New York: Thieme Medical; 2007. pp. 265-85.

[2] Kimonis V, Gold JA, Hoffman TL, Panchal J, Boyadjiev SA. Genetics of craniosynostosis. Semin Pediatr Neurol. 2007; 14: $150-61$. 
[3] Wilkie AO, Patey SJ, Kan SH, van den Ouweland AM, Hamel BC. FGFs, their receptors, and human limb malformations: clinical and molecular correlations. Am J Med Genetics. 2002; 112: $266-78$.

[4] Cohen MM., Jr. Craniosynostosis: diagnosis, evaluation and management. In: Cohen MM Jr, MacLean RE, editors. New York: Oxford University Press; 2000. pp. 158-71.

[5] Kreiborg S. Postnatal growth and development of the craniofacial complex in premature craniosynostosis. In: Cohen MM Jr, MacLean RE, editors. Craniosynostosis: diagnosis, evaluation and management. Oxford University Press: New York; 2000. pp. 158-70.

[6] Boulet SL, Rasmussen SA, Honein MA. A population-based study of craniosynostosis in metropolitan Atlanta, 1989-2003. Am J Med Genet. 2008; 146: 984-91.

[7] Kirmi O, Lo SJ, Johnson D, Anslow P. Craniosynostosis: a radiological and surgical perspective. Semin Ultrasound CT MR. 2009; 30: 492-512.

[8] Aviv RI, Rodger E, Hall CM. Craniosynostosis. Clin Radiol. 2002; 57: 93-102].

[9] Kotrikova B, Krempien R, Freier K, Mühling J. Diagnostic imaging in the management of craniosynostoses. Eur Radiol. 2007; 17: 1968-78.

[10] Kabbani H, Raghuveer TS. Craniosynostosis. Am Fam Physician. 2004; 69: 2863-70.

[11] Ridgway EB, Weiner HL. Skull deformities. Pediatr Clin North Am. 2004; 51: 359-87.

[12] Lemire R. Embryology of the skull. In: Cohen MM Jr, MacLean RE, editors. Craniosynostosis: diagnosis, evaluation and management. New York: Oxford University Press; 2000. pp. 24-32.
[13] Vannier MW. Radiologic evaluation of craniosynostosis. In: Cohen MM Jr, MacLean RE, editors. Craniosynostosis: diagnosis, evaluation and management. New York: Oxford University Press; 2000. pp. 147-56.

[14] Bridges SJ, Chambers TL, Pople IK. Plagiocephaly and head binding. Arch Dis Child. 2002; 86: 144-5.

[15] Cohen MM, Jr, MacLean RE. Anatomic, genetic, nosologic, diagnostic, and psychosocial considerations. In: Cohen MM Jr, MacLean RE, editors. Craniosynostosis: diagnosis, evaluation and management. New York: Oxford University Press; 2000. pp. 119-41.

[16] Blaser S. Abnormal skull shape. Pediatr Radiol. 2008; 38: 488-96.

[17] Sze RW, Hopper RA, Ghioni V, Gruss JS, Ellenbogen RG, King $\mathrm{D}$, et al. mdet diagnosis of the child with posterior plagiocephaly. AJR Am J Roentgenol. 2005; 185: 1342-6.

[18] Carinci F, Pezzetti F, Locci P, Becchetti E, Carls F, Avantaggiato A, et al. Apert and Crouzon syndromes: clinical findings, genes and extracellular matrix. J Craniofac Surg. 2005; 16 : 361-8.

[19] Cohen MM., Jr. Pfeiffer Syndrome. In: Cohen MM Jr, MacLean RE, editors. Craniosynostosis: Diagnosis, evaluation and management. New York: Oxford University Press; 2000. pp. 354-60.

[20] Yu JE, Jeong SY, Yang JA, Park MS, Kim HJ, Yoon SH. Genotypic and phenotypic analyses of Korean patients with syndromic craniosynostosis. Clin Genet. 2009; 76: 287-291.

[21] Goriely A, Lord H, Lim J, et al. Germline and somatic mosaicism for FGFR2 mutation in the mother of a cild with Crouzon syndrome: Implications for genetic testing in 'paternal age-effect' syndromes. Am J Med Genet. 2010; 152A: 2067-2073. 